\title{
Multi-TeV flaring from blazars: Markarian 421 as a case study
}

\author{
Sarira Sahu ${ }^{1, \mathrm{a}}$, Luis Salvador Miranda ${ }^{1, \mathrm{~b}}$, Subhash Rajpoot ${ }^{2, \mathrm{c}}$ \\ ${ }^{1}$ Instituto de Ciencias Nucleares, Universidad Nacional Autónoma de México, Circuito Exterior, C.U., A. Postal 70-543, \\ 04510 Mexico, DF, Mexico \\ 2 Department of Physics and Astronomy, California State University, 1250 Bellflower Boulevard, Long Beach, CA 90840, USA
}

Received: 22 April 2015 / Accepted: 25 February 2016 / Published online: 7 March 2016

(c) The Author(s) 2016. This article is published with open access at Springerlink.com

\begin{abstract}
The TeV blazar Markarian 421 underwent multi$\mathrm{TeV}$ flaring during April 2004 and simultaneously observations in the $\mathrm{X}$-ray and $\mathrm{TeV}$ energies were made. It was observed that the $\mathrm{TeV}$ outbursts had no counterparts in the lower energy range. One implication of this is that it might be an orphan flare. We show that Fermi-accelerated protons of energy $\leq 168 \mathrm{TeV}$ can interact with the low energy tail of the background synchrotron self-Compton photons in the inner region of the blazar to produce the multi-TeV flare and our results fit very well with the observed spectrum. Based on our study, we predict that the blazars with a deep valley in between the end of the synchrotron spectrum and the beginning of the SSC spectrum are possible candidates for orphan flaring. Future possible candidates for this scenario are the HBLs Mrk 501 and PG $1553+113$ objects.
\end{abstract}

\section{Introduction}

Active galactic nuclei (AGN) emit electromagnetic radiation the spectrum of which stretches from radio to gamma-rays and exhibit large luminosity variations on time scales ranging from less than an hour up to several years. A super massive black hole is believed to sit at the center of the AGN surrounded by an accretion disk in the inner region and a torus of gas cloud in the outer region. Oppositely directed relativistic jets are ejected from the AGN which are perpendicular to the accretion disk and the torus. In the framework of the unification scheme of AGN, blazars and radio galaxies are intrinsically the same objects, viewed at different angles with respect to the jet axis. When the angle between the jet and the line of sight is small they are called blazars and in contrast, for radio galaxies, the angle between the jet and the light of sight is large. Almost all AGN detected at very high

\footnotetext{
a e-mail: sarira@nucleares.unam.mx

b e-mail: luis.miranda@correo.nucleares.unam.mx

c e-mail: Subhash.Rajpoot@csulb.edu
}

energy (VHE) ( $>100 \mathrm{GeV})$ are blazars with the exception of the three objects, Centaurus A (Cen A) [1,2], M87 and NGC 1275 which are radio galaxies $[3,4]$. The spectral energy distribution (SED) of these AGN have a double peak structure in the $v-v F_{v}$ plane. In the context of the leptonic model, the low energy peak corresponds to the synchrotron radiation from a population of relativistic electrons in the jet. Although the general consensus is that the high energy peak corresponds to the synchrotron self Compton (SSC) scattering of the high energy electrons with their self-produced synchrotron photons, this result remains inconclusive for various reasons [2]. However, this leptonic model is very successful in explaining the multi wavelength emission from blazars and FR I galaxies [1-4].

Although the SSC scenario seems to work very well to explain the SED of AGN up to the second peak [5,6], difficulties arise in explaining the multi- $\mathrm{TeV}$ emission detected in Cen A [7], flares from the radio galaxy M87 [8], the flares from blazars 1ES $1959+650[9,10]$ and Markarian 421 (Mrk 421) [11]. Also the inevitable outcome of the leptonic scenario is that emission in multi- $\mathrm{TeV}$ energy has to be accompanied by a simultaneously enhanced emission in the synchrotron peak. Unfortunately the enhanced synchrotron emission was not observed in the flaring of 1ES 1959+650 in June 2002 [9] and also probably in the flaring of Mrk 421 in April 2004 [11], which implies that the SSC scenario may not be efficient enough to contribute in the multi- TeV regime.

To explain the orphan flaring of 1ES1959 + 650 a hadronic synchrotron mirror model was proposed by Böttcher [12]. In this model, the high energy protons from the jet interact with the primary synchrotron photons that have been reflected off clouds located at a few pc above the accretion disk. These photons are blue shifted in the jet frame so that there is a substantial decrease in the high energy protons to overcome the threshold for $\Delta$-resonance. Similarly a structured leptonic jet model is proposed to explain the orphan $\mathrm{TeV}$ flare [13]. 
In the hadronic models, the second bump is believed to occur due to the synchrotron emission from the ultra-high energy protons, or emission from the secondary electronpositron pairs or muons produced from the charged pion decay as a result of the interactions of the high energy protons with the background low energy photons. However, the efficiency of the process depends on two factors. One is the strength of the magnetic field in the jet and the other is the proton synchrotron cooling time which has to be smaller than the expansion time scale of the jet. Here the decay of neutral pions to $\gamma$-ray pairs can explain the high energy peak in the multi-TeV range $[14,15]$.

Previously it was shown that the above hadronic processes are inefficient to explain the multi- $\mathrm{TeV}$ emission. In order to explain the high energy peaks efficient acceleration of relativistic protons to ultra-high energies within the inner part of the jet outflow is required. At the same time the jet kinetic power has to exceed the Eddington luminosity, $L_{E d d}$, by several orders of magnitude. The hadronic model is employed by Zdziarski et al. [16] to explain the broad band spectra of radio-loud active galactic nuclei and by Cao et al. [17] to explain the TeV spectrum of the blazar 1ES 1101-232. The authors of Refs. [16,17] have shown that super Eddington luminosity in protons is required to explain the high energy peaks. This situation arises because the photon density in the jet is low. However, this problem can be circumvented if one assumes that within the jet there is a dense inner jet region [18]. The multi-TeV peak can also be produced by $p p$ interactions. It was shown earlier that normally the $p p$ process is inefficient to produce $\gamma$-rays in the blazar environment unless the jet-cloud interaction is taken into account [1921]. However, the $p p$ interactions are employed to explain the emission of gamma-rays from a population of massive stars surrounding the AGN jets [22,23]. Also emission of high energy gamma-rays by the interaction of the AGN jets with the tidally disrupted atmosphere of Red Giant is studied in $[24,25]$.

\section{Photohadronic model}

Sahu et al. [18,26,27] have employed the hadronic model to address the orphan $\mathrm{TeV}$ flaring of 1ES $1959+650$ and the multi-TeV emission from Cen A and M87. Recently this model is also used to make estimates of the neutrinos flux from the TeV-blazars and their spatial correlation with the IceCube events [28]. Here we use the same hadronic model to explain the multi-TeV orphan flaring of Mrk 421. In this model the Fermi-accelerated high energy protons interact with the SSC photons in the core region of the jet (few times the Schwarzschild radius $R_{S}$ ) to produce the $\Delta$-resonance. Subsequently the $\Delta$-resonance decays to charged and neutral pions as follows: $p+\gamma \rightarrow \Delta^{+} \rightarrow\left\{\begin{array}{l}p \pi^{0}, \\ n \pi^{+} \rightarrow n e^{+} v_{e} v_{\mu} \bar{v}_{\mu}\end{array}\right.$

The decay of neutral pions to $\mathrm{TeV}$ photons gives the multiTeV SED. Throughout our work we use natural units $c=$ $\hbar=1$. The $\pi^{0}$-decay $\mathrm{TeV}$ photon energy $E_{\gamma}$ and the target SSC photon energy $\varepsilon_{\gamma}$ in the observer frame are related as follows:

$E_{\gamma} \varepsilon_{\gamma} \simeq 0.032 \frac{\mathscr{D}^{2}}{(1+z)^{2}} \mathrm{GeV}^{2}$,

where $\mathscr{D}$ is the Doppler factor and $z$ is the redshift. The observed $\mathrm{TeV} \gamma$-ray energy and the proton energy $E_{p}$ are related through

$E_{p}=\frac{10 \Gamma}{\mathscr{D}} E_{\gamma}$,

where $\Gamma$ is the bulk Lorentz factor of the relativistic jet.

Multi-TeV emission is observed from many blazars and FR I galaxies by Cherenkov telescope arrays. These emissions are classified into non-flaring and flaring events. For the non-flaring events (e.g. emission from Cen A [1]), the injected proton spectrum is a power-law spectrum given by $\mathrm{d} N_{p} / \mathrm{d} E_{p} \propto E_{p}^{-\alpha}$ with $\alpha \geq 2$. These protons will interact with the background SSC photons having comoving number density $n_{\gamma}^{\prime}$ (henceforth' implies the jet comoving frame) and satisfying the kinematical conditions in Eqs. (2) and (3). As discussed in Ref. [18], the flaring occurs within a compact and confined volume of a smaller inner cone which is enclosed in a bigger outer cone as shown in Fig. 1. Similar internal compact jet models are also proposed to explain the fast variability of the blazars Mrk 501 and PKS 2155-304 $[29,30]$. In our case, the injected proton spectrum is a powerlaw spectrum supplemented with an exponential decay factor. We can express the injected proton spectrum for both nonflaring and flaring processes in a unified manner as [18,31]

$\frac{\mathrm{d} N_{p}}{\mathrm{~d} E_{p}} \propto E_{p}^{-\alpha} \begin{cases}1, & \text { non-flaring } \\ \mathrm{e}^{-E_{p} / E_{p, c},} & \text { flaring }\end{cases}$

where $E_{p, c}$ is the break energy for the high energy protons. Above this break energy the proton spectrum falls off very rapidly. The high energy protons will interact in the flaring region where the comoving photon number density is $n_{\gamma, f}^{\prime}$ to produce the $\Delta$-resonance. The photon density in the flaring region is much higher than the rest of the blob (non-flaring region with the photon density $n_{\gamma}^{\prime}$ ) probably due to the copious annihilation of electron-positron pairs, the splitting of photons in the magnetic field, the production of enhanced IC photons in this region and the Poynting flux dominated flow which can form from the magnetic reconnection in the strongly magnetized plasma around the base of the jet $[29,32,33]$. Here we assume that the spectrum of high energy 


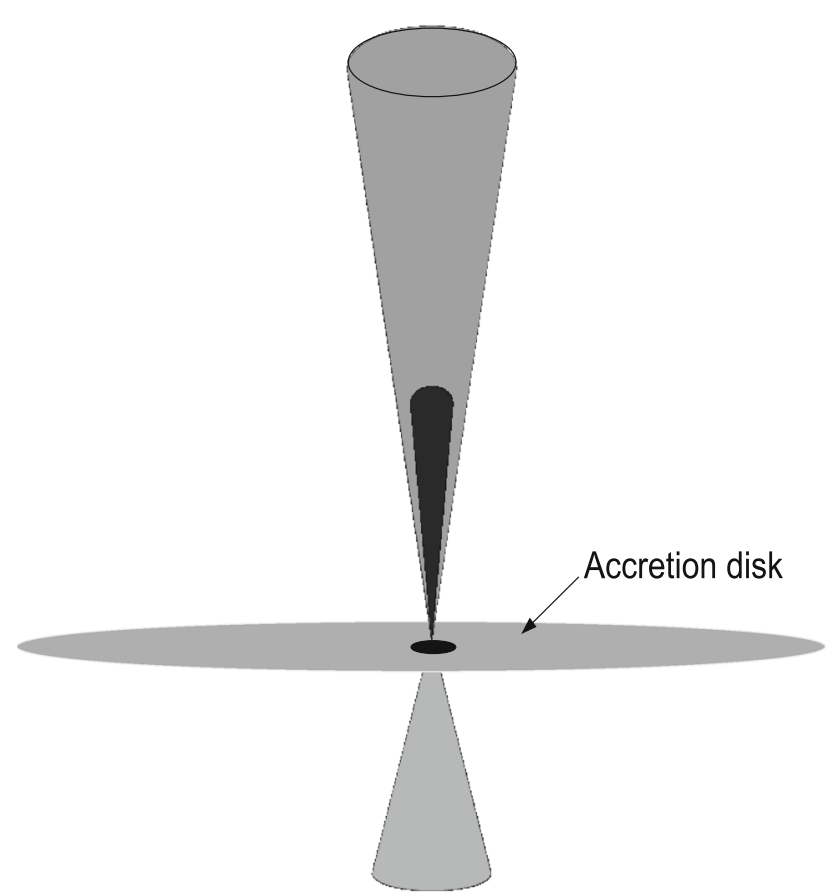

Fig. 1 Geometry of the orphan flaring of blazar Mrk 421: the hidden interior compact cone (jet) is responsible for the multi-TeV orphan flaring and the exterior cone corresponds to the normal jet

protons has a exponential decay above the $E_{p, c}$ only for the flaring case. Since the inner jet is more compact than the outer jet, the number density of photons, their energy density and the magnetic field are comparatively higher than the outer jet values. Due to higher photon density in the inner region, the multi-TeV $\gamma$-rays and neutrinos can be produced through intermediate $\Delta$-resonance as shown in Eq. (1). The optical depth of the $\Delta$-resonance process in the inner jet region is given by

$\tau_{p \gamma}=n_{\gamma, f}^{\prime} \sigma_{\Delta} R_{f}^{\prime}$

where the resonant cross section is $\sigma_{\Delta} \sim 5 \times 10^{-28} \mathrm{~cm}^{2}$. The efficiency of the $p \gamma$ process depends on the physical conditions of the interaction region, such as the size, the distance from the base of the jet, the photon density and their distribution in the region of interest.

In the inner region we compare the dynamical time scale $t_{d}^{\prime}=R_{f}^{\prime}$ with the $p \gamma$ interaction time scale $t_{p \gamma}^{\prime}=$ $\left(n_{\gamma, f}^{\prime} \sigma_{\Delta} K_{p \gamma}\right)^{-1}$ to constraint the seed photon density so that multi-TeV photons can be produced. For a moderate efficiency of this process, we can assume $t_{p \gamma}^{\prime}>t_{d}^{\prime}$ and this gives $\tau_{p \gamma}<2$, where the inelasticity parameter is assigned the usual value of $K_{p \gamma}=0.5$. Also by assuming the Eddington luminosity is equally shared by the jet and the counter jet, the luminosity within the inner region for a seed photon energy $\varepsilon_{\gamma}^{\prime}$ will satisfy $\left(4 \pi n_{\gamma, f}^{\prime} R_{f}^{\prime} \varepsilon_{\gamma}^{\prime}\right) \ll L_{\text {Edd }} / 2$. This puts an upper limit on the seed photon density as $n_{\gamma, f}^{\prime} \ll \frac{L_{\text {Edd }}}{8 \pi R_{f}^{\prime 2} \varepsilon_{\gamma}^{\prime}}$.

From Eq. (6) we can estimate the photon density in this region and this is discussed in Sect. 3. For a self consistent treatment, in principle, we should use the photon density $n_{\gamma, f}^{\prime}$ in the hidden internal jet and solve the coupled transport equations for leptons and photons along the jet axis by taking into account their respective cooling mechanisms as well as the injection spectrum of the primary particles [34]. To avoid this complication we assume the scaling behavior of the photon densities in different background energies as follows:

$\frac{n_{\gamma, f}^{\prime}\left(\varepsilon_{\gamma_{1}}^{\prime}\right)}{n_{\gamma, f}^{\prime}\left(\varepsilon_{\gamma_{2}}^{\prime}\right)}=\frac{n_{\gamma}^{\prime}\left(\varepsilon_{\gamma_{1}}^{\prime}\right)}{n_{\gamma}^{\prime}\left(\varepsilon_{\gamma_{2}}^{\prime}\right)}$

This implies that the ratio of photon densities at two different background energies $\varepsilon_{\gamma_{1}}^{\prime}$ and $\varepsilon_{\gamma_{2}}^{\prime}$ in the flaring and the nonflaring states remains the same. The photon density in the outer region is calculated from the observed flux in the usual way. In the photohadronic scenario, the number of $\pi^{0}$-decay photons at a given energy are proportional to both the number of high energy protons and the density of the SSC background photons in the jet, i.e. $N\left(E_{\gamma}\right) \propto N\left(E_{p}\right) n_{\gamma}^{\prime}$. For the flaring case $n_{\gamma}^{\prime}$ is replaced by the photon density in the flaring region $\left(n_{\gamma, f}^{\prime}\right)$. The $\gamma$-ray flux from the $\pi^{0}$ decay is deduced to be

$F_{\gamma}\left(E_{\gamma}\right) \equiv E_{\gamma}^{2} \frac{\mathrm{d} N\left(E_{\gamma}\right)}{\mathrm{d} E_{\gamma}} \propto E_{p}^{2} \frac{\mathrm{d} N\left(E_{p}\right)}{\mathrm{d} E_{p}} n_{\gamma, f}^{\prime}$.

Using the scaling behavior of Eq. (7), the observed multi$\mathrm{TeV}$ photon flux from $\pi^{0}$-decay at two different observed photon energies $E_{\gamma 1}$ and $E_{\gamma 2}$ can be expressed as

$\frac{F_{\gamma}\left(E_{\gamma_{1}}\right)}{F_{\gamma}\left(E_{\gamma_{2}}\right)}=\frac{n_{\gamma}^{\prime}\left(\varepsilon_{\gamma_{1}}\right)}{n_{\gamma}^{\prime}\left(\varepsilon_{\gamma_{2}}\right)}\left(\frac{E_{\gamma_{1}}}{E_{\gamma_{2}}}\right)^{-\alpha+2} e^{-\left(E_{\gamma_{1}}-E_{\gamma_{2}}\right) / E_{c}}$,

where $E_{\gamma_{1,2}}$ correspond to the proton energy $E_{p_{1,2}}$. In this derivation we have used the relations $E_{p_{1}} / E_{p_{2}}=E_{\gamma_{1}} / E_{\gamma_{2}}$ and $E_{p_{1,2}} / E_{p, c}=E_{\gamma_{1,2}} / E_{c}$, where $E_{c}$ is the $\gamma$-ray cut-off energy corresponding to $E_{p, c}$. By using the known flux at a particular energy in the flaring/non-flaring state, we can calculate the flux at other energies using Eq. (9). The normalization constant can also be calculated.

In terms of SSC photon energy and its luminosity, the photon number density $n_{\gamma}^{\prime}$ is expressed as

$n_{\gamma}^{\prime}\left(\varepsilon_{\gamma}\right)=\eta \frac{L_{\gamma, \mathrm{SSC}}(1+z)}{\mathscr{D}^{2+\kappa} 4 \pi R^{\prime 2} \varepsilon_{\gamma}}$

where $\eta$ is the efficiency of SSC process and $\kappa$ describes whether the jet is continuous $(\kappa=0)$ or discrete $(\kappa=1)$. In this work we take $\eta=1$ for $100 \%$ efficiency. The SSC 
photon luminosity is expressed in terms of the observed flux $\left(\Phi_{\mathrm{SSC}}\left(\varepsilon_{\gamma}\right)=\varepsilon_{\gamma}^{2} \mathrm{~d} N_{\gamma} / \mathrm{d} \varepsilon_{\gamma}\right)$ and is given by

$L_{\gamma, \mathrm{SSC}}=\frac{4 \pi \mathrm{d}_{L}^{2} \Phi_{\mathrm{SSC}}\left(\varepsilon_{\gamma}\right)}{(1+z)^{2}}$.

Furthermore, by using Eq. (2), we can simplify the ratio of photon densities given in Eq. (7) to

$\frac{n_{\gamma}^{\prime}\left(\varepsilon_{\gamma_{1}}\right)}{n_{\gamma}^{\prime}\left(\varepsilon_{\gamma_{2}}\right)}=\frac{\Phi_{\mathrm{SSC}}\left(\varepsilon_{\gamma_{1}}\right)}{\Phi_{\mathrm{SSC}}\left(\varepsilon_{\gamma_{2}}\right)} \frac{E_{\gamma_{1}}}{E_{\gamma_{2}}}$

Here we take $\kappa=0$ as representative value for the calculation of $n_{\gamma}^{\prime}$. We note that $\kappa=1$ will only lead to the suppression of the photon number density for the outer enveloping jet without changing the main conclusions of our work. This follows from Eq. (12) where proportionality factors depending on $\kappa$ cancel out. Equation (9) in terms of observed SSC flux and $E_{\gamma}$ takes the following form:

$\frac{F_{\gamma}\left(E_{\gamma_{1}}\right)}{F_{\gamma}\left(E_{\gamma_{2}}\right)}=\frac{\Phi_{\operatorname{SSC}}\left(\varepsilon_{\gamma_{1}}\right)}{\Phi_{\operatorname{SSC}}\left(\varepsilon_{\gamma_{2}}\right)}\left(\frac{E_{\gamma_{1}}}{E_{\gamma_{2}}}\right)^{-\alpha+3} \mathrm{e}^{-\left(E_{\gamma_{1}}-E_{\gamma_{2}}\right) / E_{c}}$,

and it uses the SED of SSC photon calculated using the leptonic model. Here the multi- TeV flux is proportional to $E_{\gamma}^{-\alpha+3}$ and $\Phi_{\mathrm{SSC}}\left(\varepsilon_{\gamma}\right)$, while in Eq. (9) it is proportional to $E_{\gamma}^{-\alpha+2}$ and to the photon number density $n_{\gamma}^{\prime}\left(\varepsilon_{\gamma}\right)$. In the photohadronic process $(p \gamma)$, the multi-TeV photon flux is expressed as

$F\left(E_{\gamma}\right)=A_{\gamma} \Phi_{\operatorname{SSC}}\left(\varepsilon_{\gamma}\right)\left(\frac{E_{\gamma}}{\mathrm{TeV}}\right)^{-\alpha+3} \mathrm{e}^{-E_{\gamma} / E_{c}}$.

Both $\varepsilon_{\gamma}$ and $E_{\gamma}$ satisfy the condition given in Eq. (2) and the dimensionless constant $A_{\gamma}$ is given by

$A_{\gamma}=\left(\frac{F\left(E_{\gamma_{2}}\right)}{\Phi_{\mathrm{SSC}}\left(\varepsilon_{\gamma_{2}}\right)}\right)\left(\frac{\mathrm{TeV}}{E_{\gamma_{2}}}\right)^{-\alpha+3} \mathrm{e}^{E_{\gamma_{2}} / E_{c}}$.

Equation (14) will be used to calculate the multi-TeV flux from both non-flaring (without exponential decay term) and flaring events from AGN and their subclasses if the emission is due to photohadronic process from the core region. For different blazars/AGN, the value of $A_{\gamma}$ will be different. This is discussed in the next section. We can calculate the Fermiaccelerated high energy proton flux $F_{p}$ from the $\mathrm{TeV} \gamma$-ray flux through the relation

$F_{p}\left(E_{p}\right)=7.5 \times \frac{F_{\gamma}\left(E_{\gamma}\right)}{\tau_{p \gamma}\left(E_{p}\right)}$.

The optical depth $\tau_{p \gamma}$ is given in Eq. (5). For the observed highest energy proton energy $E_{p}, F_{p}\left(E_{p}\right)$ will always be smaller than the Eddington flux $F_{\text {Edd }}$. This condition puts a lower limit on the optical depth of the process and is given by

$\tau_{p \gamma}\left(E_{p}\right)>7.5 \times \frac{F_{\gamma}\left(E_{\gamma}\right)}{F_{E d d}}$.

From the comparison of different time scales and from Eq. (17) we will be able to constraint the seed photon density in the inner jet region.

\section{Mrk 421}

Mrk 421 is a high synchrotron peaked BL Lac object (HBL) and was the first extragalactic source with a redshift of $z=0.031$ to be established as a $\mathrm{TeV}$ emitter [35]. It has a luminosity distance $d_{L}$ of about $129.8 \mathrm{Mpc}$. Its central supermassive black hole is assumed to have a mass $M_{B H} \simeq(2-9) \times 10^{8} M_{\odot}$ corresponding to a Schwarzschild radius of $(0.6-2.7) \times 10^{14} \mathrm{~cm}$ and the Eddington luminosity $L_{\text {Edd }}=(2.5-11.3) \times 10^{46} \mathrm{erg} \mathrm{s}^{-1}$. The synchrotron peak of its SED is in the soft to medium X-ray range and the $\mathrm{SSC}$ peak is in the $\mathrm{GeV}$ range. It is one of the fastest varying $\gamma$-ray sources. In the past, through dedicated multi wavelength observations, the source has been studied intensively. These studies show a correlation between $\mathrm{X}$-rays and very high energy (VHE) $\gamma$-rays. A one-zone SSC model explains the observed SED reasonably well [36]. Several major flares were observed in the 2003/2004 season. During April 2004, a large flare took place both in the $\mathrm{X}$-rays and the $\mathrm{TeV}$ energy bands. The flare lasted for more than two weeks (from MJD 53,104 to roughly MJD 53,120). But due to a large data gap between MJD 53,093 and 53,104, it is difficult to exactly quantify the duration. The source was observed simultaneously at $\mathrm{TeV}$ energies with the Whipple $10 \mathrm{~m}$ telescope and at X-ray energies with the Rossi X-ray timing explorer (RXTE). It was also observed simultaneously at radio and optical wavelengths. During the flaring it was observed that the $\mathrm{TeV}$ flares had no coincident counterparts at longer wavelengths. Also it was observed that the X-ray flux reached its peak 1.5 days before the $\mathrm{TeV}$ flux did during this outburst. Generally it is believed that the $\mathrm{TeV}$ flare might not be a true orphan flare, like the one observed in 1ES 1959+650. On the other hand remarkable similarities between the orphan $\mathrm{TeV}$ flare in 1ES 1959+650 and Mrk 421 were observed, including similar variation patterns in the $\mathrm{X}$-ray spectrum.

By using the one-zone SSC model, the average SED of Mrk 421 is fitted in Fig. 11 of Ref. [11]. In Fig. 2 we show the SED of Mrk 421. In this figure, the red crosses to the extreme left are the measurements in the radio frequency band measured by the $26 \mathrm{~m}$ telescope at the University of Michigan Radio Astronomy Observatory (UMRAO) and by the $13.7 \mathrm{~m}$ 


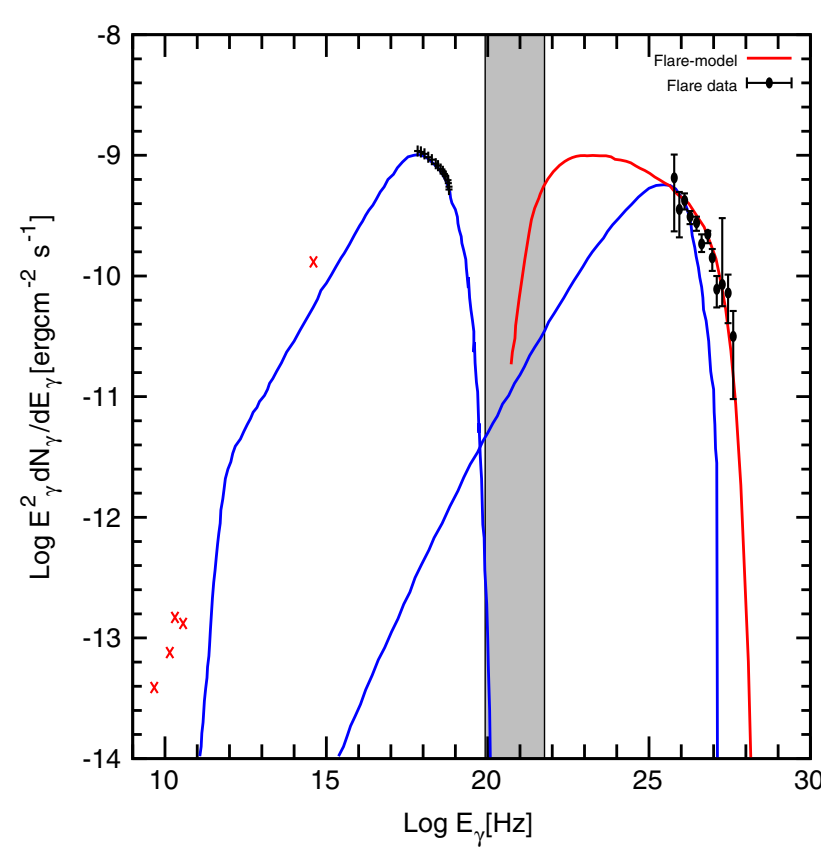

Fig. 2 The SED of Mrk 421 is shown in all the energy bands which are taken from Ref. [11]. The flare of April 2004 in multi-TeV energy is shown here. The hadronic model fit to the April 2004 data is shown as continuous line to the extreme right. The shaded region is the energy range of SSC photons where the Fermi-accelerated protons collide to produce the $\Delta$-resonance

Metsähovi radio telescope at Helsinki. The single cross (red) in the optical range is measured by Fred Lawrence Observatory (FLWO) $1.2 \mathrm{~m}$ telescope. The points of the first peak in the X-ray range are from Rossi X-ray Explorer (RXTE) [11].

In the one-zone leptonic model, the blob of size $R_{b}^{\prime} \sim$ $0.7 \times 10^{16} \mathrm{~cm}$ moves down the conical jet with a Lorentz factor $\Gamma \simeq 14$ and a Doppler factor of $\mathscr{D}=14$. The emitting region is filled with an isotropic electron population and a randomly oriented magnetic field $B^{\prime}=0.26 \mathrm{G}$. In the present work we study the flaring of Mrk 421 during April 2004. We use the parameters of the one-zone leptonic model of ref. [11]. The parameters of the one-zone synchrotron model are summarized in Table 1. In principle the Lorentz factor in the inner jet should be larger than the outer jet. But here for simplicity we assume that $\Gamma_{\text {out }} \simeq \Gamma_{\text {in }} \simeq \Gamma$.

\section{Results}

The flaring of Mrk 421 in April 2004 was observed in the energy range $0.25 \mathrm{TeV}\left(6.0 \times 10^{25} \mathrm{~Hz}\right) \leq E_{\gamma} \leq$ $16.85 \mathrm{TeV}\left(4.1 \times 10^{27} \mathrm{~Hz}\right)$ by the Whipple telescope. In the hadronic model alluded to previously, this corresponds to the Fermi-accelerated proton energy in the range $2.5 \mathrm{TeV} \leq$ $E_{p} \leq 168 \mathrm{TeV}$ and the corresponding background photon energy will lie in the range $23.6 \mathrm{MeV}\left(5.7 \times 10^{21} \mathrm{~Hz}\right) \geq$
Table 1 These parameters (up to $B^{\prime}$ ) are taken from the one-zone synchrotron model of Ref. [11] which are used to fit the SED of Mrk 421. The last three parameters are obtained from the best fit to the observed flare data in our model

\begin{tabular}{lll}
\hline Parameter & Description & Value \\
\hline$M_{\mathrm{BH}}$ & Black hole mass & $(2-9) \times 10^{8} M_{\odot}$ \\
$\mathrm{z}$ & Redshift & 0.031 \\
$\Gamma$ & Bulk Lorentz factor & 14 \\
$\mathscr{D}$ & Doppler factor & 14 \\
$R_{b}^{\prime}$ & Blob radius & $0.7 \times 10^{16} \mathrm{~cm}$ \\
$B^{\prime}$ & Magnetic field & $0.26 \mathrm{G}$ \\
$R_{f}^{\prime}$ & Inner blob radius & $3 \times 10^{15} \mathrm{~cm}$ \\
$\alpha$ & Spectral index & 2.7 \\
$E_{c}$ & $\gamma$-Ray cut-off energy & $6.2 \mathrm{TeV}$ \\
\hline
\end{tabular}

$\varepsilon_{\gamma} \geq 0.35 \mathrm{MeV}\left(8.4 \times 10^{19} \mathrm{~Hz}\right)$. This range of $\varepsilon_{\gamma}$ is the shaded region shown in Fig. 2 which is in the low energy tail of the SSC photons. For the calculation of the normalized multi-TeV flux we take into account one of the observed $\mathrm{TeV}$ fluxes from the flare at its corresponding energy. With the use of Eq. (13) we calculate other TeV fluxes. In this model the free parameters are the spectral index $\alpha$ and the $\mathrm{TeV} \gamma$-ray cut-off energy $E_{c}$. The best fit is obtained for the values $\alpha=2.7$ and $E_{c}=6.2 \mathrm{TeV}$. This value of $E_{c}$ corresponds to the proton cut-off energy $E_{p, c}=62 \mathrm{TeV}$ and the SSC photon energy $\varepsilon_{\gamma, S S C}=0.96 \mathrm{MeV}\left(2.3 \times 10^{20} \mathrm{~Hz}\right)$ which is very close to the beginning of the SSC energy as shown in Fig. 2. This shows that for orphan flaring, the cutoff energy $E_{c}$ is due to the change from the synchrotron band to the SSC band and can be calculated from their crossover energy region.

In Fig. 3 we show the observed multi-TeV SED and the predictions of our model. In our results, the presence of $\Phi_{\mathrm{SSC}}\left(\varepsilon_{\gamma}\right)$ in Eq. (14) modifies the power-law with the exponential fall-off scenario. From the best fit parameters we obtain the value of the dimensionless constant $A_{\gamma} \simeq 20$ in Eq. (15). By using the same best fit parameters, we have also calculated the value of $A_{\gamma}$ from the multi-TeV flare of 1ES $1959+650$ and M87 and the multi-TeV emission from Centaurus A, which are determined to be 86,2 and $\mathscr{O}\left(10^{-3}\right)$, respectively. We observe that for orphan flaring the condition $A_{\gamma} \gg 1$ is satisfied as is seen from 1ES 1959+650 and Mrk421. On the other hand for non-orphan flaring $A_{\gamma} \leq 1$.

In the flaring state, as has been alluded to before, in general, the flux of the two opposing jets can be as high as $F_{\text {Edd }} / 2$. However, the highest energy protons with $E_{p}=168$ $\mathrm{TeV}$ must have flux $F_{p}<F_{\text {Edd }} / 2$. Using Eq. (16) this constraint translates into $\tau_{p \gamma}>0.02$, which corresponds to $n_{\gamma, f}^{\prime}>1.3 \times 10^{10} \mathrm{~cm}^{-3}$ in the inner jet. However, the hidden jet lies between $R_{s}$ and $R_{b}^{\prime}$. As one representative value 


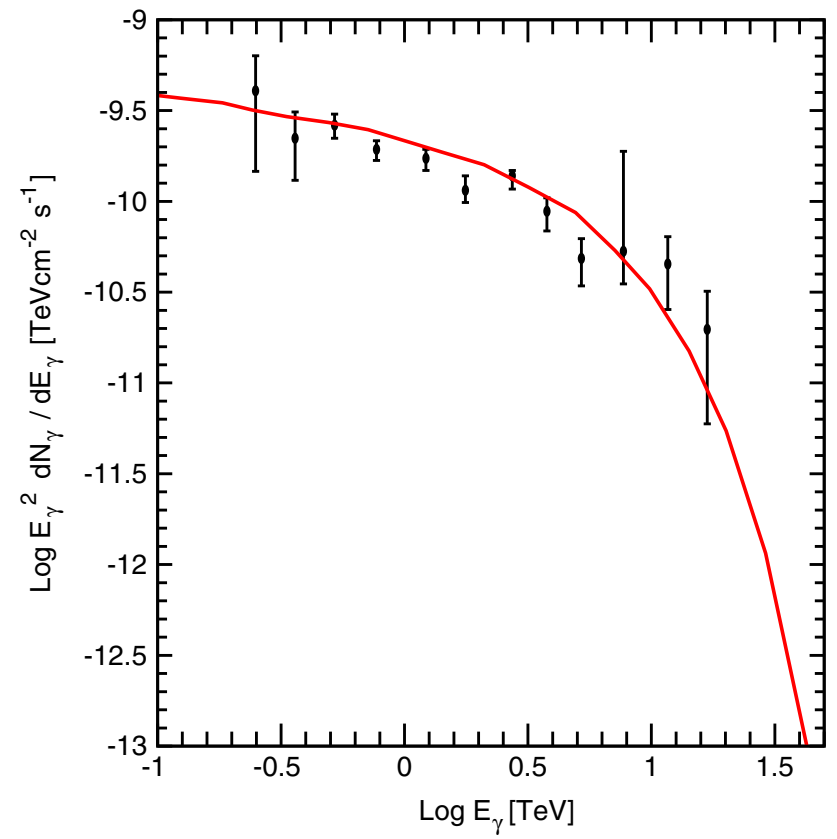

Fig. 3 The continuous curve is the hadronic model fit to the multi-TeV flaring data of Mrk 421

we take $R_{f}^{\prime} \simeq 3 \times 10^{15}$. This value of $R_{f}^{\prime}$ corresponds to a day scale variability, which follows from the fact that the flaring lasted for more than two weeks. From Eq. (6) the seed photon density for $\varepsilon_{\gamma}=0.35 \mathrm{MeV}$ satisfies the inequality $n_{\gamma, f}^{\prime}<8.9 \times 10^{10} \mathrm{~cm}^{-3}$, which translates to the optical depth to be constrained as $\tau_{p \gamma}<0.13$. The upshot of all this is that, while the value of $\tau_{p \gamma}<2$ is a generic constraint, a more refined present analysis constrains the optical depth to lie in the range $0.02<\tau_{p \gamma}<0.13$. From this the constraint on the photon number density in the inner jet region is determined to be $1.3 \times 10^{10} \mathrm{~cm}^{-3}<n_{\gamma, f}^{\prime}<8.9 \times 10^{10} \mathrm{~cm}^{-3}$, which shows that the photon density in this region is high. Due to the adiabatic expansion of the inner blob, the photon density will be reduced to $n_{\gamma}^{\prime}$ and the energy will dissipate once these photons cross into the bigger outer cone. In spite of the two-zones structure, only the outer zone will be responsible for the observed synchrotron and the IC peaks. From the leptonic model fit to the SED, the magnetic field in the outer jet region is determined to be $B^{\prime}=0.26 \mathrm{G}$. In general, higher values of the magnetic field are expected to be present in the inner jet region. The maximum proton energy in the hidden jet region will be $E_{p, \max } \sim 10^{18}\left(B_{f}^{\prime} / 1 G\right)$ $\mathrm{eV}$ and for larger magnetic fields, $E_{p, \max }$ can even be higher.

In comparison, in the non-flaring jet scenario case we estimate the proton flux needed to explain the observed $\mathrm{TeV} \gamma$-rays as follows: Corresponding to the seed photon energy $\varepsilon_{\gamma}=0.35 \mathrm{MeV}$, the photon density and the optical depth are, respectively, $n_{\gamma}^{\prime} \sim 4 \times 10^{3} \mathrm{~cm}^{-3}$ and $\tau_{p \gamma} \sim 1.4 \times 10^{-8}$. This corresponds to the observed $\mathrm{TeV}$ photon energy $E_{\gamma}=16.8 \mathrm{TeV}$ and the proton energy $E_{p}=168$, respectively. The high energy $\gamma$-ray flux for $E_{\gamma}=16.8$ is $F_{\gamma} \sim 3.16 \times 10^{-11} \mathrm{erg} \mathrm{cm}^{-2} \mathrm{~s}^{-1}$. Using Eq. (16) we obtain the high energy proton flux as $F_{p} \simeq 10^{6} \times F_{\text {Edd }}$. This shows that the normal jet model needs super Eddington power in the protons to explain the high energy peak [16], whereas our inner jet scenario eliminates this extreme energy requirement.

In passing we note that, in the energy range considered, the high energy protons will be accompanied by high energy electrons. These electrons will emit synchrotron photons in the energy range $4 \times 10^{19} \mathrm{~Hz}$ to $2 \times 10^{23} \mathrm{~Hz}$ when encountering the magnetic field of the jet. These energy range photons lie in the lower part of the SSC spectrum and thus will not be observed due to their flux being low. The high energy electrons will also emit SSC photons and their energy is given by $E_{\mathrm{IC}} \sim \gamma_{\mathrm{e}}^{2} \varepsilon_{\text {syn }}$. By considering the electron Lorentz factor in the range $7 \times 10^{2} \leq \gamma_{\mathrm{e}} \leq 4 \times 10^{4}$ [36] and the peak energy of the synchrotron photons to be $\varepsilon_{\text {syn }} \sim 10^{18} \mathrm{~Hz}$, we determine the SSC process contribution to the flux in the energy range $2 \mathrm{GeV} \leq E_{\mathrm{IC}} \leq 6.6 \mathrm{TeV}$. The details of the SSC flux depend on the breaks in SSC spectrum and the spectral index. It is observed that during the flaring of Mrk 421, the X-ray emission reached the peak days after the $\mathrm{TeV}$ emission, which poses a serious challenge to the SSC model [11].

The multi-TeV photons in the energy range $0.25 \mathrm{TeV} \leq$ $E_{\gamma} \leq 16.8 \mathrm{TeV}$ can interact with the background photons to produce $\mathrm{e}^{+} \mathrm{e}^{-}$pairs in which the individual electrons or positrons have energy $E_{\gamma} / 2$. To produce the $\mathrm{e}^{+} \mathrm{e}^{-}$pair, the required threshold seed photon energy of $\varepsilon_{\gamma} \geq 2 m_{\mathrm{e}}^{2} / E_{\gamma}$ is needed. During the flaring, multi-TeV $\gamma$-rays will interact with soft seed photons in the energy range $0.05 \mathrm{eV} \leq$ $\varepsilon_{\gamma} \leq 3.5 \mathrm{eV}$ (in between the infrared and the visible range), where $\sigma_{\gamma \gamma} \sim 1.7 \times 10^{-25} \mathrm{~cm}^{2}$ is the maximum cross section. For higher $\varepsilon_{\gamma}, \sigma_{\gamma \gamma}$ will be smaller. We consider the sources of these soft photons to be from the synchrotron emission of $1-10 \mathrm{GeV}$ electrons interacting with the magnetic field $B \sim 1$ Gauss and the flux of the ambient photons coming from the disk. On the other hand the multi- $\mathrm{TeV} \gamma$-rays are produced beyond this region where the photons make the low energy tail (0.35-23.5 MeV range) of the IC photons. The regions $0.05-3.5 \mathrm{eV}$ and $0.35-23.5 \mathrm{MeV}$ represent two distinct energy ranges. The $\mathrm{TeV}$ photons will mostly encounter the tail region of the SSC spectrum. The pair production cross section for $\varepsilon_{\gamma} \geq 0.35 \mathrm{MeV}$ is very small $\sigma_{\gamma \gamma} \leq 10^{-30} \mathrm{~cm}^{2}$, which corresponds to a mean free path of $\lambda_{\gamma \gamma} \geq 10^{19} \mathrm{~cm}$. Hence, the $\mathrm{TeV}$ photons will not be attenuated much due to the $\mathrm{e}^{+} \mathrm{e}^{-}$pair production. Also it has been observed that, during the flaring of Mrk 421, the variation in the light curves at optical and radio wavelengths are minimal [11], which implies that the low energy photon production was sup- 
pressed. In addition to the above scenario, the positrons produced from the $\pi^{+}$decay have energy $E_{\gamma} / 2$ and will radiate synchrotron photons in the energy range $2 \times 10^{17}$ to $9 \times 10^{20}$ $\mathrm{Hz}$. The photon flux $F_{\mathrm{e}^{+} \text {,syn }}$ from the synchrotron radiation of $\mathrm{e}^{+}$will be much smaller than $F_{\gamma}\left(E_{\gamma}=0.25 \mathrm{TeV}\right) / 8$, i.e., $F_{\mathrm{e}^{+} \text {,syn }} \ll 8 \times 10^{-11} \mathrm{erg} \mathrm{cm}^{-2} \mathrm{~s}^{-1}$. This flux is well below the observed flux limit in the normal case as can be seen from Fig. 2. From the above analysis our conclusion is that the photon fluxes from the synchrotron emission of the electrons and positrons are not observable during the flaring event of Mrk 421 in April 2004. This situation is very much like the one discussed in the orphan flaring of 1ES $1959+650$. Hence we conclude that the flaring of Mrk 421 is orphan in nature. In principle, the flux of multi- $\mathrm{TeV} \gamma$-rays from the extragalactic sources is attenuated due to interactions with the diffuse extragalactic background light (EBL) through the process $\gamma_{\mathrm{TeV}}+\gamma_{\mathrm{b}} \rightarrow \mathrm{e}^{+} \mathrm{e}^{-}$due to the energy dependent optical depth [37-39]. But for low redshifts and the energy range of our interest, the optical depth $\tau_{\gamma \gamma}$ does not vary much. Hence we can assume an almost constant optical depth [31] so that the spectral shape remains nearly unchanged.

Our study shows that the flaring of blazars can be explained through photohadronic interactions in a compact and confined region within the blazar jet where the photon density is high. In particular, from the study of Mrk 421, we deduce that the orphan flaring can only be possible for those blazars which have a deep valley in between the end of the synchrotron SED and the beginning of the SSC SED as shown in Fig. 2. We note that the HBLs Mrk 501 and PG $1553+113$ are possible candidates for orphan flaring in the future. For the orphan flaring events we find $A_{\gamma} \gg 1$ and for non-orphan flaring $A_{\gamma} \leq 1$.

If Mrk 501 were to produce an orphan flare then the flare energy will lie in the range $1 \mathrm{TeV} \leq E_{\gamma} \leq 8.6 \mathrm{TeV}$ and this corresponds to the background SSC photon energy in the range $4.3 \mathrm{MeV} \geq \varepsilon_{\gamma} \geq 0.5 \mathrm{MeV}$. We have estimated this by taking the parameters of Mrk 501 from Ref. [40] as follows: $\mathscr{D}=12, z=0.034$. In this case the maximum Fermiaccelerated proton energy will be $E_{p} \leq 10 E_{\gamma} \sim 86 \mathrm{TeV}$. Also, another condition which must be satisfied for orphan flaring is

$$
A_{\gamma}=\frac{F(8.6 \mathrm{TeV})}{\Phi_{\mathrm{SSC}}(0.5 \mathrm{MeV})}\left(\frac{1}{8.6}\right)^{-\alpha+3} \mathrm{e}^{8.6 /\left(E_{c} / \mathrm{TeV}\right)} \gg 1
$$

The value of $\Phi_{\text {SSC }}(0.5 \mathrm{MeV})$ will be obtained from the SED of Mrk 501. It is to be noted that, if $\mathscr{D}$ changes, accordingly the values of $E_{\gamma}, \varepsilon_{\gamma}$ and the $E_{p}$ will also change. However, in our scenario the condition of Eq. (18) is independent of these changes.

\section{Conclusions}

We conclude that the orphan flaring of Mrk 421 can be explained well by the photohadronic model. We observe that, in this model, the multi-TeV photon flux is proportional to $\Phi_{\mathrm{SSC}}\left(\varepsilon_{\gamma}\right) E_{\gamma}^{-\alpha+3}$ supplemented with an exponential decay term as shown in Eq. (14). This implies that the Fermiaccelerated protons interact with the background photons (in the low energy tail) of the SSC spectrum. In the present work we have shown that the higher photon density in the internal jet scenario eliminates the requirement of the super Eddington luminosity to explain the multi-TeV emission. During the April 2004 flaring of Mrk 421, we have demonstrated that the flux from the synchrotron emission from the high energy $\mathrm{e}^{+}$and $\mathrm{e}^{-}$is suppressed relative to the normal flux, implying that the flaring was orphan in nature, like the one observed in 1ES 1959+650. We have also considered the type of blazar spectrum that will result in orphan flaring and predict that the Mrk 501 and the PG 1553+113 are possible candidates for orphan flaring in the future. Monitoring of these objects by the $\mathrm{TeV}$ gamma-ray telescopes will shed more light on the details of the orphan flaring mechanism.

We wish to thank the anonymous referee for helpful comments. The work of S.S. is partially supported by DGAPAUNAM (Mexico) Project No. IN110815.

Open Access This article is distributed under the terms of the Creative Commons Attribution 4.0 International License (http://creativecomm ons.org/licenses/by/4.0/), which permits unrestricted use, distribution, and reproduction in any medium, provided you give appropriate credit to the original author(s) and the source, provide a link to the Creative Commons license, and indicate if changes were made. Funded by SCOAP $^{3}$.

\section{References}

1. A.A. Abdo et al., Fermi LAT Collaboration. Astrophys. J. 719, 1433-1444 (2010). arXiv:1006.5463 [astro-ph.HE]

2. P. Roustazadeh, M. Böttcher, Astrophys. J. 728, 134 (2011)

3. G. Fossati, L. Maraschi, A. Celotti, A. Comastri, G. Ghisellini, Mon. Not. Roy. Astron. Soc. 299, 433 (1998). arXiv:astro-ph/9804103

4. G. Ghisellini, A. Celotti, G. Fossati, L. Maraschi, A. Comastri, Mon. Not. Roy. Astron. Soc. 301, 451 (1998). arXiv:astro-ph/9807317

5. C.D. Dermer, R. Schlickeiser, Astrophys. J. 416, 458 (1993)

6. M. Sikora, M.C. Begelman, M.J. Rees, Astrophys. J. 421, 153 (1994)

7. F. Aharonian et al. [HESS Collaboration], Astrophys. J. 695, L40 (2009). arXiv:0903.1582 [astro-ph.CO]

8. A. Abramowski et al., [H.E.S.S. and VERITAS Collaborations], Astrophys. J. 746, 151 (2012). arXiv:1111.5341 [astro-ph.CO]

9. H. Krawczynski, S.B. Hughes, D. Horan, F. Aharonian, M.F. Aller, H. Aller, P. Boltwood, J. Buckley et al., Astrophys. J. 601, 151 (2004). arXiv:astro-ph/0310158 
10. W. Cui et al., VERITAS Collaboration. AIP Conf. Proc. 745, 455 (2005). arXiv:astro-ph/0410160

11. M. Blazejowski, G. Blaylock, I.H. Bond, S.M. Bradbury, J.H. Buckley, D.A. Carter-Lewis, O. Celik, P. Cogan et al., Astrophys. J. 630, 130 (2005). arXiv:astro-ph/0505325

12. M. Böttcher, Astrophys. J. 621, 176 (2005) [Erratum-ibid. 641, 1233 (2006)]. arXiv:astro-ph/0411248

13. M. Kusunose, F. Takahara, Astrophys. J. 651, 113 (2006). arXiv:astro-ph/0607063

14. A. Mucke, J.P. Rachen, R. Engel, R.J. Protheroe, T. Stanev, Publ. Astron. Soc. Austral. 16, 160 (1999)

15. A. Mucke, R.J. Protheroe, Astropart. Phys. 15, 121 (2001)

16. A.A. Zdziarski, M. Böttcher, Mon. Not. Roy. Astron. Soc. 450(1), L21 (2015)

17. G. Cao, J. Wang, Astrophys. J. 783, 108 (2014). arXiv:1401.3970 [astro-ph.HE]

18. S. Sahu, A.F.O. Oliveros, J.C. Sanabria, Phys. Rev. D 87, 103015 (2013)

19. A. Atoyan, C.D. Dermer, Phys. Rev. Lett. 87, 221102 (2001)

20. A.M. Atoyan, C.D. Dermer, Astrophys. J. 586, 79 (2003). arXiv:astro-ph/0209231

21. C.D. Dermer, K. Murase, H. Takami, Astrophys. J. 755, 147 (2012). arXiv:1203.6544 [astro-ph.HE]

22. W. Bednarek, R.J. Protheroe, Mon. Not. Roy. Astron. Soc. 287, L9 (1997). arXiv:astro-ph/9612073

23. A. Araudo, V. Bosch-Ramon, G.E. Romero, Mon. Not. Roy. Astron. Soc. 436, 3626 (2013). arXiv:1309.7114 [astro-ph.HE]

24. V. Bosch-Ramon, M. Perucho, M.V. Barkov, Astron. Astrophys. 539, A69 (2012). arXiv:1201.5279 [astro-ph.HE]

25. M.V. Barkov, F.A. Aharonian, S.V. Bogovalov, S.R. Kelner, D. Khangulyan, Astrophys. J. 749, 119 (2012). arXiv:1012.1787 [astro-ph.HE]

26. S. Sahu, B. Zhang, N. Fraija, Phys. Rev. D 85, 043012 (2012). arXiv:1201.4191 [astro-ph.HE]
27. S. Sahu, E. Palacios, Eur. Phys. J. C 75(2), 52 (2015). arXiv:1310.1381 [astro-ph.HE]

28. S. Sahu, L.S. Miranda, Eur. Phys. J. C 75(6), 273 (2015). arXiv:1408.3664 [astro-ph.HE]

29. D. Giannios, D.A. Uzdensky, M.C. Begelman, Mon. Not. Roy. Astron. Soc. 395, L29 (2009). arXiv:astro-ph/9807317. arXiv:0901.1877 [astro-ph.HE]

30. G. Ghisellini, F. Tavecchio, G. Bodo, A. Celotti, Mon. Not. Roy. Astron. Soc. 393, 16 (2009). arXiv:0810.5555 [astro-ph]

31. F. Aharonian et al., HEGRA Collaboration. Astron. Astrophys. 406, L9 (2003). arXiv:astro-ph/0305275

32. M. Kachelriess, S. Ostapchenko, R. Tomas, Publ. Astron. Soc. Austral. 27, 482 (2010). arXiv:1002.4874 [astro-ph.HE]

33. D. Giannios, D.A. Uzdensky, M.C. Begelman, Mon. Not. Roy. Astron. Soc. 402, 1649 (2010). arXiv:0907.5005 [astro-ph.HE]

34. M.M. Reynoso, G.E. Romero, M.C. Medina, Astron. Astrophys. 545, A125 (2012). arXiv:1208.5284 [astro-ph.HE]

35. M. Punch, C.W. Akerlof, M.F. Cawley, M. Chantell, D.J. Fegan, S. Fennell, J.A. Gaidos, J. Hagan et al., Nature 358, 477 (1992)

36. A.A. Abdo et al., MAGIC Collaboration. Astrophys. J. 736, 131 (2011)

37. J.D. Finke, S. Razzaque, C.D. Dermer, Astrophys. J. 712, 238 (2010). arXiv:0905.1115 [astro-ph.HE]

38. A. Dominguez, J.R. Primack, D.J. Rosario, F. Prada, R.C. Gilmore, S.M. Faber, D.C. Koo, R.S. Somerville et al., Mon. Not. Roy. Astron. Soc. 410, 2556 (2011). arXiv:1007.1459 [astro-ph.CO]

39. Y. Inoue, S. Inoue, M.A.R. Kobayashi, R. Makiya, Y. Niino, T. Totani, Astrophys. J. 768, 197 (2013). arXiv:1212.1683 [astroph.CO]

40. B. Bartoli et al., ARGO-YBJ Collaboration. Astrophys. J. 758, 2 (2012). arXiv:1209.0534 [astro-ph.HE] 